\title{
Kernresonanz-Emissionslinien während rascher Radikalreaktionen
}

\author{
II. Chemisch induzierte dynamische Kernpolarisation \\ J. BARgON und H. Fischer \\ Deutsches Kunststoff-Institut Darmstadt \\ (Z. Naturforschg. 22 a, 1556-1562 [1967] ; eingegangen am 16. Juni 1967)
}

\begin{abstract}
Previously observed nuclear magnetic resonance emission lines of products of rapid radical reactions are explained by chemically induced dynamic nuclear polarization. This polarization occurs after the production of radicals by the dynamic coupling between radical electrons and protons, and it is transfered to the products by the secondary reaction. A theoretical analysis shows that this concept leads to a correct order of magnitude calculation of the intensity of emission. New experiments are described the results of which support the explanation. It is concluded that chemically induced dynamic nuclear polarization is not restricted to the systems treated so far but may occur in other investigations also.
\end{abstract}

In dieser Arbeit wird eine Deutung der von BARGON $^{1}$ entdeckten Protonenresonanzemissionslinien von Produkten in einem Magnetfeld ablaufender Radikalreaktionen vorgeschlagen. Die Emissionslinien werden auf eine dynamische Polarisation der Spins der Radikalprotonen zurückgeführt, die mit der Erzeugung der Radikale einsetzt und durch die Weiterreaktion der Radikale auf die Protonen der Produkte übertragen wird.

Dieser Vorschlag wird in Abschnitt 1 dieser Arbeit erläutert und in Abschnitt 2 für ein einfaches Modellsystem eingehender behandelt. Dabei werden Gleichungen entwickelt, die die Intensität der Emissionssignale bei stationärem Reaktionsablauf beschreiben können. In Abschnitt 3 wird dann über Untersuchungen zur Abhängigkeit der Emissionsintensität beim Dibenzoylperoxidzerfall ${ }^{1}$ von der Meßfrequenz und von der Konzentration paramagnetischer Zusatzstoffe berichtet, die die vorgeschlagene Deutung stützen. Abschnitt 4 behandelt schließlich einen qualitativen Vergleich zwischen Theorie und Experiment, und in Abschnitt 5 werden einige Folgerungen angegeben.

\section{Das Konzept}

In der vorausgehenden Arbeit ${ }^{1}$ wurde gezeigt, daß die Emissionslinien Molekeln $M$ zugehören, die bei radikalischen Zerfalls- und Übertragungsreaktionen in einem äußeren Magnetfeld entstehen. Diese

1 J. B ARgon, H. Fischer u. U. Johnsen, Z. Naturforschg. 22 a, 1551 [1967].

2 A. A. Frost u. R. G. Pearson, Kinetics and Mechanism, J. Wiley, New York 1961.
Reaktionen werden hier zu

$$
\begin{aligned}
& R-R \rightarrow 2 R^{\prime}, \\
& R^{\cdot}+L \rightarrow M+L^{\prime}
\end{aligned}
$$

verallgemeinert.

Danach zerfällt eine Verbindung $\mathrm{R}-\mathrm{R}$ in zwei Radikale $\mathrm{R}^{*}$, die anschließend mit einer anderen Verbindung $\mathrm{L}$ zu den Molekeln M und Sekundärradikalen $\mathrm{L}^{\circ}$ weiterreagieren. Die Radikale $\mathrm{R}^{\circ}$ und die Molekeln M sollen dabei Protonen enthalten, wie es der Beobachtung ${ }^{1}$ entspricht.

Die Emissionslinien führen wir nun auf eine dynamische Kernpolarisation der Protonen in den Radikalen $\mathrm{R}^{\cdot}$ zurück, die jedoch an den Molekeln M beobachtet wird. Dazu verwenden wir die bekannte Deutung von Emissionslinien beim OverhauserEffekt an Radikallösungen ${ }^{3,4}$, die wir durch Einschluß der Reaktionen (1) und (2) abändern. Dazu setzen wir voraus :

1. Bei der Reaktion (1) werden die beiden Elektronenspin-ZeEman-Niveaus der Radikale $\mathrm{R}^{*}$ gleich besetzt.

2. Bei der Reaktion (2) bleibt die momentane Einstellung der Protonenspins der Radikale R' im äußeren Magnetfeld erhalten und wird auf die Molekeln M übertragen.

3. Es existiert eine zeitlich statistisch schwankende magnetische Kopplung zwischen den Elektronenspins und den Protonenspins der Radikale R'.

3 A. Abragam, Principles of Nuclear Magnetism, Clarendon Press, Oxford 1961.

${ }^{4}$ K. H. Hausser u. D. Stehlik, Dynamic Nuclear Polarization im Liquids, Advan. Magnetic. Res. Bd. 3 [1967], Acad. Press, im Druck. 
Die erste dieser Voraussetzungen entspricht dem Prinzip der Spinerhaltung bei chemischen Reaktionen, da bei (1) in einem äußeren Magnetfeld aus einer Verbindung $\mathrm{R}-\mathrm{R}$ mit Singulettgrundzustand zwei Radikale mit Dublettgrundzuständen entstehen. Die zweite Voraussetzung erscheint plausibel, da der Reaktionsschritt (2) in einer Zeit erfolgt (Stoßzeit ${ }^{2}$ von $R^{\cdot}$ und $L: \approx 10^{-11}$ bis $10^{-13} \mathrm{sec}$ ), die wesentlich kleiner ist als die reziproke Resonanzfrequenz der Protonen $\left(v^{-1} \approx 10^{-7}\right.$ bis $\left.10^{-8} \mathrm{sec}\right)$. Die dritte ist bei Radikalen durch die Wechselwirkung der magnetischen Momente von Radikalelektron und Radikalprotonen gegeben, wobei insbesondere die $\mathrm{Di}$ pol-Dipol-Wechselwirkung durch den rotatorischen Anteil der BRowsschen Molekularbewegung zeitlich statistisch moduliert ist.

Mit diesen Voraussetzungen sind dann die Emissionslinien leicht zu erklären:

Bei der Radikalentstehung wird eine Gleichbesetzung der Elektronenspin-ZeEman-Niveaus gebildet. Diese sucht sich in die dem thermischen Gleichgewicht entsprechende BoltzmanN-Verteilung durch Spin-Gitter-Relaxation infolge der dynamischen Kopplungen der magnetischen Momente der Radikalelektronen mit magnetischen Momenten ihrer molekularen Umgebung umzuwandeln, zu denen auch die Momente der Radikalprotonen gehören. Mit den dabei auftretenden Relaxationsübergängen der Elektronen sind Relaxationsübergänge der Protonenspins der Radikale verknüpft, wodurch die energetisch höheren Zeeman-Niveaus dieser Protonenspins intermediär stärker besetzt werden als die energetisch niederen Niveaus. Reagieren nun die Radikale vor der endgültigen Einstellung des Gleichgewichts nach Reaktion (2) zu Molekeln weiter, so kann die intermediär vorhandene Überbesetzung der energetisch höheren Protonenspinniveaus auf die Molekeln übertragen werden. Die Molekeln besitzen damit kurz nach ihrer Entstehung eine negative Spinpolarisation der Protonen, die zur intermediären induzierten Emission der Übergangsfrequenz der Protonenresonanz führt.

Dieses Konzept entspricht der Deutung des OverHAUSER-Effekts an Radikallösungen ${ }^{3,4}$. Im einzelnen gehören hier die dynamisch gekoppelten Elektronenund Kernspins zur selben Radikalmolekel, so daß eine dynamische Kernpolarisation von Protonen in

5 D. Stehlik u. K. H. Hausser, Z. Naturforschg. 22 a, 914 [1967].
Radikalen vorliegt, wie sie erstmals von STEHLIK und HAUSSER $^{5}$ beobachtet wurde. Weiter enthält unser Konzept die Übertragung einer in Radikalen induzierten dynamischen Kernpolarisation auf Molekeln (Voraussetzung 2.), die an anderen Systemen bereits von Richards und Mitarbeitern ${ }^{6}$ gefunden wurde. Im Gegensatz zu dem bisher bekannten wird jedoch hier die Gleichbesetzung der Elektronenspinniveaus durch eine chemische Reaktion und nicht durch Hochfrequenzeinstrahlung der Elektronenspinresonanzfrequenz erreicht. Wir bezeichnen den Vorgang deshalb als „chemisch induzierte dynamische Kernpolarisation".

\section{Theoretische Behandlung}

Zur quantitativen Behandlung nehmen wir ein einfaches Modell an, in dem sowohl die Radikale $\mathrm{R}^{\text {. }}$ als auch die Molekeln M nur jeweils ein (und dasselbe) Proton enthalten. Zwischen den magnetischen Momenten von Radikalelektron- und -proton bestehe nur die durch die Brownsche Molekularbewegung modulierte Dipol-Dipol-Kopplung, deren zeitlicher Mittelwert verschwindet. Diesem Modell entspricht das in Abb. 1 dargestellte statische Schema der Energieniveaus von $\mathrm{R}^{\circ}$ und $\mathrm{M}$ im Magnetfeld.

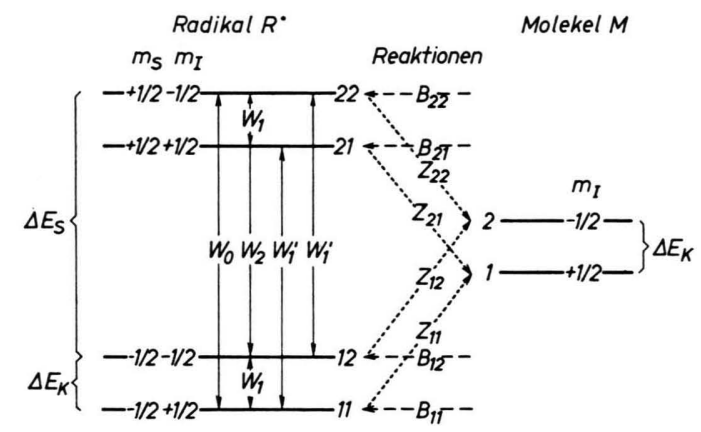

Abb. 1. Energieniveaus von $\mathrm{R}^{\cdot}$ und $\mathrm{M}$ und ihre Besetzungen durch die Reaktionen. $\Delta E_{\mathrm{s}} / \Delta E_{\mathrm{K}}=\hbar \gamma_{\mathrm{s}} H / \hbar \gamma_{\mathrm{p}} H=657$.

Wir bezeichnen die Besetzungszahlen der Niveaus $i k$ der Radikale mit $N_{i k}$, die der Niveaus $k$ der Molekeln mit $n_{k}$. Die Gesamtzahl der Radikale sei $N$, der Molekeln $n$. Weiterhin führen wir die Größen

$$
\begin{aligned}
& \Delta N_{\mathrm{s}}=\left(N_{11}+N_{12}\right)-\left(N_{21}+N_{22}\right), \\
& \Delta N_{\mathrm{p}}=\left(N_{11}+N_{21}\right)-\left(N_{12}+N_{22}\right)
\end{aligned}
$$

und

$$
\Delta n=n_{1}-n_{2}
$$

${ }^{6}$ R. A. Dwek, J. G. Kenworthy u. R. E. Richards, Mol. Phys. 10, 529 [1966]. 
ein, denen bei entsprechenden Experimenten das Elektronenspinresonanzsignal der Radikale R;, das Protonenresonanzsignal der Radikale $\mathrm{R}^{*}$ und das Protonenresonanzsignal der Molekeln M proportional sind. Durch die Reaktion (1) sollen nun die Radikale $\mathrm{R}^{\cdot}$ mit den Bildungsgeschwindigkeiten $B_{i k}$ in den Niveaus erzeugt werden (vgl. Abb. l). Wir nehmen dabei an, daß alle $B_{i k}$ gleich

$$
B_{i k}=\frac{1}{4} B
$$

sind. (Im Prinzip sollten die $B_{i k}$ wegen der nichtverschwindenden Polarisation des Protonenspinsystems der Molekeln R-R vor der Reaktion voneinander verschieden sein. Wird das berücksichtigt, so ändern sich die im folgenden abgeleiteten Formeln nur unwesentlich.) Durch Reaktion (2) verschwinden dann die Radikale $\mathrm{R}^{*}$ unter Bildung der Molekeln aus den Niveaus mit den Zerfallsgeschwindigkeiten

$$
Z_{i k}=N_{i k} / \tau,
$$

wobei $\tau$ die mittlere Lebensdauer der Radikale ist. Dann gilt für die Gesamtzahl der Radikale

$$
\mathrm{d} N / \mathrm{d} t=B-\mathrm{N} / \tau .
$$

Nehmen wir nun an ${ }^{3}$, daß die Elektronenspins der Radikale durch die zeitlich veränderliche Dipol-Dipol-Kopplung mit den Protonen und durch eine Kopplung an andere magnetische Momente des Gitters relaxieren und daß die Protonenspins der Radikale nur durch die zeitlich veränderliche DipolDipol-Kopplung mit den Elektronen relaxieren, so lassen sich durch Betrachtung der zeitlichen Änderungen der Besetzungszahlen $N_{i k}$ (vgl. Abb. 1) für $\triangle N_{\mathrm{s}}$ und $N_{\mathrm{p}}$ folgende Ausdrücke ableiten:

$$
\begin{array}{r}
\frac{\mathrm{d}}{\mathrm{d} t}\left(\Delta N_{\mathrm{s}}\right)=-\frac{\Delta N_{\mathrm{s}}}{\tau}-\varrho^{\prime \prime}\left(\Delta N_{\mathrm{s}}-N \Delta E_{\mathrm{s}} / 2 k T\right) \\
+\sigma\left(\Delta N_{\mathrm{p}}-N \Delta E_{\mathrm{K}} / 2 k T\right) \\
\frac{\mathrm{d}}{\mathrm{d} t}\left(\Delta N_{\mathrm{p}}\right)=-\frac{\Delta N_{\mathrm{p}}}{\tau}-\varrho\left(\Delta N_{\mathrm{p}}-N \Delta E_{\mathrm{K}} / 2 k T\right) \\
+\sigma\left(\Delta N_{\mathrm{s}}-N \Delta E_{\mathrm{s}} / 2 k T\right) .
\end{array}
$$

Dabei sind

$$
\begin{aligned}
& \varrho=w_{0}+2 w_{1}+w_{2}, \\
& \varrho^{\prime \prime}=\varrho^{\prime}+w_{8}=w_{0}+2 w_{1}{ }^{\prime}+w_{2}+w_{\mathrm{s}}, \\
& \sigma=w_{2}-w_{0},
\end{aligned}
$$

wobei $w_{0}, w_{1}, w_{1}{ }^{\prime}$ und $w_{2}$ die durch die ElektronProton-Kopplung bewirkten Relaxationsübergangswahrscheinlichkeiten sind (vgl. Abb. 1) und $w_{\mathrm{s}}$ alle anderen Relaxationsübergangswahrscheinlichkeiten des Elektronenspins einschließt. Für $w_{0}, w_{1}, w_{1}{ }^{\prime}, w_{2}$ gelten ferner die Beziehungen (3)

$$
\begin{aligned}
& w_{0}=\frac{1}{10} \gamma_{\mathrm{p}}^{2} \gamma_{\mathrm{s}}^{2} \hbar^{2}\left(\tau_{\mathrm{c}} / b^{6}\right) /\left[1+\left(\omega_{\mathrm{s}}+\omega_{\mathrm{p}}\right)^{2} \tau_{\mathrm{c}}^{2}\right], \\
& w_{1}=\frac{3}{20} \gamma_{\mathrm{p}}^{2} \gamma_{\mathrm{s}}^{2} \hbar^{2}\left(\tau_{\mathrm{c}} / b^{6}\right) /\left[1+\omega_{\mathrm{p}}{ }^{2} \tau_{\mathrm{c}}^{2}\right], \\
& w_{1}^{\prime}=\frac{3}{20} \gamma_{\mathrm{p}}^{2} \gamma_{\mathrm{s}}^{2} \hbar^{2}\left(\tau_{\mathrm{c}} / b^{6}\right) /\left[1+\omega_{\mathrm{s}}^{2} \tau_{\mathrm{c}}{ }^{2}\right], \\
& w_{2}=\frac{6}{10} \gamma_{\mathrm{p}}^{2} \gamma_{\mathrm{s}}^{2} \hbar^{2}\left(\tau_{\mathrm{c}} / b^{6}\right) /\left[1+\left(\omega_{\mathrm{s}}-\omega_{\mathrm{p}}\right)^{2} \tau_{\mathrm{c}}{ }^{2}\right]
\end{aligned}
$$

unter der Annahme, daß die Dipol-Dipol-Kopplung durch den rotatorischen Anteil der Brownschen Molekularbewegung statistisch moduliert ist und daß sie allein die Relaxation der Protonenspins hervorruft. In (7) sind dabei $\gamma_{\mathrm{p}}$ und $\gamma_{\mathrm{s}}$ die gyromagnetischen Verhältnisse von Proton und Elektron, $\omega_{\mathrm{p}}$ und $\omega_{\mathrm{s}}$ ihre Resonanzkreisfrequenzen im äußeren Magnetfeld $H, \tau_{\mathrm{c}}$ ist die rotatorische Korrelationszeit der Brownschen Bewegung und $b$ der mittlere Abstand zwischen Elektron und Proton.

Die Gleichungen (4) und (5) stellen SolomonGleichungen eines Zwei-Spin-Systems dar ${ }^{3,}$, die zur Berücksichtigung der Reaktion erweitert wurden. Die Einzelterme sind dabei nicht wie häufig üblich ${ }^{3,4,7}$ durch Elektronen- und Kernspinpolarisationen, sondern durch Besetzungszahldifferenzen ausgedrückt. Dies ist jedoch hier vorteilhaft, da die in die Polarisation eingehende Gesamtspinzahl $N$ in unserem Fall zeitlich nicht konstant ist.

Aus den gekoppelten Differentialgleichungen (3), (4) und (5) können nun die Größen $N(t), \Delta N_{\mathrm{s}}(t)$ und $\Delta N_{\mathrm{p}}(t)$ berechnet werden, wenn $B(t), \tau, w_{\mathrm{s}}$ und $w_{i}$ bekannt sind. Daraus folgen die Intensitäten der Úbergangslinien der Radikale $\mathrm{R}^{*}$ bei Protonen- oder Elektronenspinresonanzexperimenten.

Für die hier besonders interessierenden Molekeln $\mathrm{M}$ gelten weiter die Beziehungen

und

$$
\mathrm{d} n / \mathrm{d} t=N / \tau
$$

$$
\frac{\mathrm{d}(\Delta n)}{\mathrm{d} t}=\frac{\Delta N_{\mathrm{p}}}{\tau}-\frac{1}{T_{1 \mathrm{M}}}\left(\Delta n-n \cdot \frac{\Delta E_{\mathrm{K}}}{2 k T}\right) .
$$

Sie liefern bei bekannter Spin-Gitter-Relaxationszeit $T_{1 \mathrm{M}}$ der Molekeln und aus (3), (4) und (5) berechneten $\Delta N_{\mathrm{p}}(t)$ die Größe $\Delta n(t)$ und damit die Intensität des Protonenresonanzsignals der Molekeln während der Reaktion.

- Aus den hier in allgemeiner Form angeschriebenen Formeln (3) bis (9) läßt sich nur schwer ablesen, daß $\Delta n(t)$ intermediär negativ werden, das Protonenresonanzsignal also in Emission auftreten kann. Dies wird deutlicher, wenn eine stationäre

\footnotetext{
7 I. Solomon, Phys. Rev. 99, 559 [1955].
} 
Phase der Reaktionen und Relaxationsfolge existiert, gilt. In diesem Fall folgt aus (3) bis (9) :

für die

$$
N=B \cdot \tau
$$$$
\frac{\mathrm{d} N}{\mathrm{~d} t}=\frac{\mathrm{d}\left(\Delta N_{\mathrm{s}}\right)}{\mathrm{d} t}=\frac{\mathrm{d}\left(\Delta N_{\mathrm{p}}\right)}{\mathrm{d} t}=\frac{\mathrm{d}(\Delta n)}{\mathrm{d} t}=0
$$

Diese Gleichungen lassen sich nun einfach diskutieren: Zunächst kann durch Einsetzen der Parameter $\varrho^{\prime \prime}, \varrho$ und $\sigma$ aus den Gln. (6) und (7) gezeigt werden, $\mathrm{da}$ stets

$$
\left(1+\varrho^{\prime \prime} \tau\right)(1+\varrho \tau)-\sigma^{2} \tau^{2}>0
$$

ist. Da alle anderen in den Gleichungen auftretenden Ausdrücke positiv sind, folgt damit unmittelbar (12), daß ein Elektronenspinresonanzsignal der Radikale $\mathrm{R}^{*}$ bei einer Radikalkonzentration $N$ während der Radikalreaktion gemessen kleiner ist, als ein bei gleicher Konzentration ohne Reaktion gefundenes Signal, das

$$
N \cdot \Delta E_{\mathrm{s}} / 2 k T
$$

proportional ist. Je kürzer die Lebensdauer $\tau$ ist, desto kleiner ist das Signal. Es verschwindet im Extremfall $\tau \rightarrow 0$. Dies gilt auch für das Protonenresonanzsignal der Radikale.

Das Protonenresonanzsignal der Molekeln weicht während der Reaktion um den zweiten Term der Gl. (14) von dem bei gleicher Molekelkonzentration $n$ ohne Reaktion beobachteten Signal ab. Definieren wir einen Verstärkungsfaktor durch die Reaktion in der bei der Behandlung der dynamischen Kernpolarisation üblichen Weise als:

$$
V=\frac{\Delta n-n \cdot\left(\Delta E_{\mathrm{K}} / 2 k T\right)}{n \cdot\left(\Delta E_{\mathrm{K}} / 2 k T\right)},
$$

so folgt aus (14)

$$
V=\frac{N}{n} \frac{T_{1 \mathrm{M}}}{\tau}\left(1-\frac{\left(1+\varrho^{\prime \prime} \tau\right)+\sigma \tau\left(\Delta E_{\mathrm{s}} / \Delta E_{\mathrm{K}}\right)}{\left(1+\varrho^{\prime \prime} \tau\right)(1+\varrho \tau)-\sigma^{2} \tau^{2}}\right) .
$$

Wird $V<-1$, so erscheint das Protonenresonanzsignal in Emission. Bei Beachtung der in (15) auftretenden Vorzeichen und der Beziehung ${ }^{3}$

$$
\Delta E_{\mathrm{s}} / \Delta E_{\mathrm{K}}=657
$$

erscheint dies für günstige Werte der in (15) eingehenden Größen durchaus möglich. In Abschnitt 4 dieser Arbeit wird eine Abschätzung des nach dieser Gleichung zu erwartenden Verstärkungsfaktors durchgeführt. Sie zeigt, daß Gl. (15) den Effekt in der Größenordnung richtig beschreibt.

Darüberhinaus liefert (15) die Möglichkeit, die Gültigkeit unseres Deutungsvorschlags experimentell $\mathrm{zu}$ überprüfen. Aus (6) und (7) geht hervor, daß $\varrho \prime$, $\varrho$ und $\sigma$ mit steigenden Resonanzkreisfrequenzen $\omega_{\mathrm{s}}$ und $\omega_{\mathrm{p}}$, also mit steigendem äußeren Magnetfeld, sinken. Mit (15) sollte damit die Emissionsintensität, bei gleichem Reaktionsablauf aber in verschiedenen äußeren Magnetfeldern gemessen, mit steigendem Magnetfeld abfallen. Weiterhin hängt $V$ nach (15) von der Spin-Gitter-Relaxationszeit $T_{1 \mathrm{M}}$ ab. Verkürzt man diese z. B. durch Zugabe von paramagnetischen Ionen zu den reagierenden Lösungen, so sollte die Emissionsintensität bei gleichem Reaktionsverlauf mit steigender Ionenkonzentration ebenfalls sinken. Im folgenden Abschnitt werden die entsprechenden Experimente behandelt. Ihre Ergebnisse zeigen, daß die aus (15) gewonnenen Voraussagen zutreffen.

\section{Experimentelle Ergebnisse}

Zur Prüfung der Gültigkeit der in den Abschnitten 1 und 2 angegebenen Überlegungen und Formeln werden einige Untersuchungen an dem bereits früher behandelten ${ }^{1}$ System Dibenzoylperoxid in Cyclohexanon bei einer Zerfallstemperatur von $110{ }^{\circ} \mathrm{C}$ durchgeführt. Dieses System ist sicher wesentlich komplizierter als das in Abschnitt 2 analysierte Modell, da weder die Radikale R' (hier Benzoyloxy- und Phenylradikale $\mathrm{C}_{6} \mathrm{H}_{5}{ }^{\circ}$ ), noch die Molekeln $\mathrm{M}$ (hier Benzol $\mathrm{C}_{6} \mathrm{H}_{6}$ ) nur ein Proton enthalten. 
Außerdem sind die Protonen in den Radikalen mit den Elektronen außer dipolar auch skalar gekoppelt. Da die dipolare Kopplung jedoch sicher wesentlich zu den Relaxationen beiträgt, sollte unsere Deutung der Emissionslinien auch auf dieses System qualitativ übertragen werden können.

Im einzelnen wurden wie früher ${ }^{1}$ vorbereitete Lösungen in den vorgewärmten Meßkopf der Spektrometer gebracht. Die Amplitude des Protonenresonanzsignals der gebildeten Benzolmolekeln wurde während des Ablaufs der Reaktionen mit der beschriebenen Aufnahmetechnik ${ }^{1}$ verfolgt. Die Temperatur der Lösungen stieg in allen Fällen in etwa 60 Sekunden auf die Spektrometertemperatur $\left(110{ }^{\circ} \mathrm{C}\right)$ an.

In einer ersten Meßreihe wurden Lösungen fester Dibenzoylperoxidkonzentration $\quad\left(5 \cdot 10^{-2}\right.$ Mol Dibenzoylperoxid pro Mol Cyclohexanon) eingesetzt, denen paramagnetisches Eisen(III) -chlorid $\left(\mathrm{FeCl}_{3}\right.$, wasserfrei, E. M e r ck AG, Darmstadt) in verschiedenen Konzentrationen vor der Reaktion zugesetzt wurde. Abb. 2 zeigt den Verlauf der Amplitude des Benzolresonanzsignals der einzelnen Lösungen als Funktion der Zeit. Dabei sind die hier durchgezeichneten Kurven als Einhüllende der Meßkurven nach Abb. 3 der vorausgehenden Arbeit gewonnen worden. Als Parameter ist die Eisen(III)-Konzentration in Mol-\% angegeben.

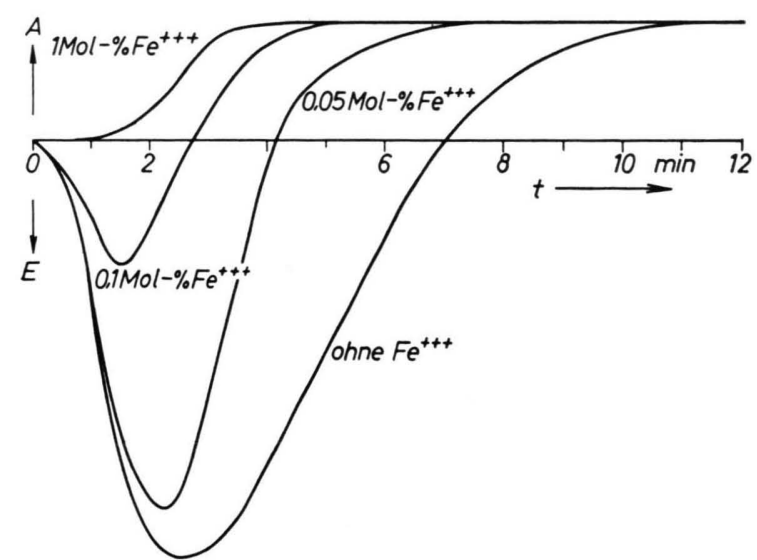

Abb. 2. Der Einfluß paramagnetischer Zusätze auf den Amplitudenverlauf der Benzolresonanzlinien. 5 Mol-\% BPO in Cyclohexan, $110^{\circ} \mathrm{C}, 56,4 \mathrm{MHz}$.

Bei allen Lösungen ist, wie auch gaschromatographische Messungen zeigten, am Ende der Reaktion, hier nach etwa 12 Minuten, ungefähr gleich viel Benzol gebildet. Der paramagnetische Zusatz beeinflußt also die Reaktionenfolge nicht. Der Verlauf der Amplitude zu Beginn der Reaktion hängt jedoch stark von der Eisen(III)-Konzentration ab. Mit steigender Eisen(III)-Konzentration sinkt die Emissionsintensität, und das Emissionsmaximum verschiebt sich nach kürzeren Zeiten. Bei einer Konzentration von $10^{-2} \mathrm{Mol} \mathrm{FeCl}_{3}$ pro Mol Cyclohexanon tritt überhaupt keine Emission mehr auf, so daß die hier gemessene Kurve die Bildung des Benzols als Funktion der Zeit wiedergibt. Wie führen diese Befunde auf eine Verkürzung der Relaxationszeiten zurück und werten sie nach Abschnitt 2 als Stütze für unseren Deutungsvorschlag.

In einer zweiten Meßreihe wurden Lösungen ebenfalls fester Dibenzoylperoxidkonzentration eingesetzt. Das Resonanzsignal des Benzols wurde jetzt jedoch bei den drei verschiedenen Protonenresonanzfrequenzen $40,0 \mathrm{MHz}, 56,4 \mathrm{MHz}$ und $100,0 \mathrm{MHz}$ verfolgt. Aus Abb. 3 geht hervor, daß die Emissionsintensität mit steigender Meßfrequenz und damit steigender Magnetfeldstärke sinkt, wie es nach Ab-

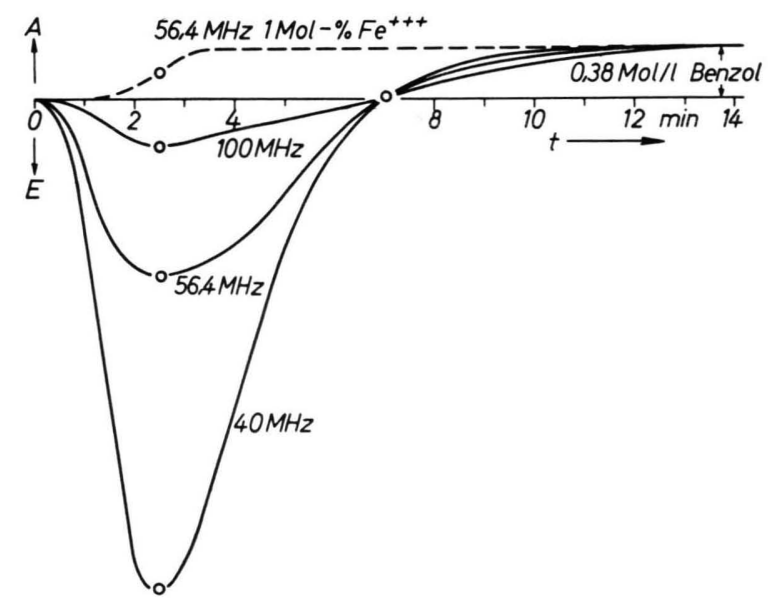

Abb. 3. Abhängigkeit des Amplitudenverlaufs der Benzolresonanzlinie von der Meßfrequenz. $5 \mathrm{Mol}-\%$ BPO in Cyclohexanon, $110^{\circ} \mathrm{C}$; Meßfrequenzen 40, 56,4 und $100 \mathrm{MHz}$.

schnitt $2 \mathrm{zu}$ erwarten war. Dieser Befund wurde auch bei anderen Ausgangskonzentrationen von Dibenzoylperoxid erhalten.

Aus Abb. 3 dassen sich nun durch Vergleich der Emissionsintensitäten mit den Amplituden der Benzolresonanz ohne Emission, deren Verlauf gestrichelt eingezeichnet ist (vgl. auch Abb. 2), Verstärkungsfaktoren ablesen, die den in Abschnitt 2 definierten entsprechen. Die Reaktion durchläuft nämlich bei Erreichen des Emissionsmaximums kurzzei- 
tig eine stationäre Phase, so daß sich $V$ experimentell als

$$
V=\frac{\text { Maximales Emissionssignal }- \text { Benzolsignal ohne Emission }}{\text { Benzolsignal ohne Emission }}
$$

definieren und aus den in Abb. 3 angegebenen ausgezeichneten Punkten ablesen läßt. Die so gemessenen Verstärkungsfaktoren sind $V=-20$ bei $40 \mathrm{MHz}$, $V=-8,5$ bei $56,4 \mathrm{MHz}$ und $V=-2,9$ bei $100 \mathrm{MHz}$.

\section{Abschätzung des Verstärkungsfaktors beim Dibenzoylperoxidzerfall}

Die experimentell bestimmten Verstärkungsfaktoren werden in diesem Abschnitt mit Werten verglichen, die aus der Gleichung (15) folgen, wobei angenommen wird, daß diese einfache Gleichung auf das hier behandelte System angewendet werden darf. Dies ist sicher nur als sehr grobe Näherung erlaubt, doch sollten die theoretischen Werte in der Größenordnung mit den beobachteten übereinstimmen. Zunächst werden die in (15) eingehenden Parameter $N=B \tau, n=\int B \mathrm{~d} t, T_{1 \mathrm{M}}, \tau, \varrho^{\prime \prime}, \varrho$ und $\sigma$ angegeben oder abgeschätzt.

a) $B$ und $\int B \mathrm{~d} t$ können als Bildungsgeschwindigkeit des Benzols zum Zeitpunkt maximaler Emissionsintensität und als die zu diesem Zeitpunkt bereits gebildete Benzolmenge prinzipiell aus den Kurven der Benzolbildung (Abb. 2, 3) gewonnen werden. Aus dem bisher vorliegenden experimentellen Material schätzen wir $B$ zu etwa $5 \cdot 10^{-3}$ bis $10^{-2}$ $\mathrm{Mol} / l \cdot \sec$ und $\int B \mathrm{~d} t$ zu etwa 0,15 bis $0,25 \mathrm{Mol} / l \mathrm{ab}$.

b) $T_{1 \mathrm{M}}$, die Spin-Gitter-Relaxationszeit der Benzolmolekeln, sollte nach unserer Meinung etwa 5 bis $10 \mathrm{sec}$ betragen. Sie ist wegen den in unseren Systemen wahrscheinlich auftretenden paramagnetischen Verunreinigungen, wie den Radikalen $\mathrm{R}^{\prime}$ oder gelösten Sauerstoffmolekeln, sicher kleiner als die Spin-Gitter-Relaxationszeit von reinem Benzol bei Zimmertemperatur, die in ${ }^{9}$ zu $T_{1 \mathrm{M}}=19 \mathrm{sec}$ angegeben wird. Andererseits ist sie vermutlich größer als die beobachtete Halbwertsbreite der Benzolresonanzlinie von etwa 5 sec.

c) Die mittlere Lebensdauer $\tau$ der Radikale wird durch Reaktion (2) bestimmt und durch die Be-

8 J. E. Bennett, B. Mile u. A. Thomas, Proc. Roy. Soc. London A 293, 246 [1966].

9 A. I. Zhernovor u. G. D. Latyshew, Nuclear Magnetic Resonance in a Flowing Liquid, Consultants Bureau, New York, nach Atomiizdat, Moskau 1965. ziehung ${ }^{2}$

$$
\tau^{-1}=\frac{1}{R} \cdot \frac{\mathrm{d} R}{\mathrm{~d} t}=k \cdot[\mathrm{L}]
$$

beschrieben. Dabei ist [L] die Konzentration der Molekeln L, mit denen die Radikale reagieren (2), in unserem Fall des Cyclohexanons, und $k$ ist die Geschwindigkeitskonstante der Reaktion. Nach unserem Wissen ist $k$ für die Reaktion von Phenylradikalen mit Cyclohexanon bisher unbekannt. Eine im Prinzip ähnliche Reaktion von Phenylradikalen mit Acetophenon ist jedoch von Duncan und TrotmanDickenson ${ }^{10}$ in der Gasphase untersucht worden. Übertragen wir ihre Ergebnisse für $k$ auf unsere Reaktion, so folgt $k \approx 10^{5} \mathrm{l} / \mathrm{Mol} \cdot \mathrm{sec}$. Da L bei unseren Experimenten $[\mathrm{L}]=10 \mathrm{Mol} / l$ war, folgt $\tau \approx$ $10^{-6}$ sec. Dieser Wert stellt nach unserer Meinung eine untere Grenze für $\tau$ dar, da Reaktionen in Lösung vermutlich langsamer als in der Gasphase ablaufen ${ }^{2}$. Eine obere Grenze für $\tau$ erhalten wir aus der Beziehung (10)

$$
N=B \cdot \tau \text {. }
$$

ESR-Untersuchungen an den reagierenden Lösungen zeigten, daß die mittlere Radikalkonzentration $N$ stets geringer war als die Nachweisgrenze des ESRSpektrometers, die etwa $10^{-6} \mathrm{Mol} / l$ betrug. Mit $N<10^{-6} \mathrm{Mol} / l$ und $B \sim 10^{-2} \mathrm{Mol} / l \cdot \sec$ folgt also $\tau<10^{-4}$ sec. Damit sollte $\tau$ im Bereich von etwa $10^{-4}$ bis $10^{-6}$ sec liegen.

d) Die Größen $\varrho \prime$, $\varrho$ und $\sigma$ werden durch die Gln. (6) und (7) auf die Relaxationswahrscheinlichkeit $w_{\mathrm{s}}$ der Elektronen, die rotatorische Korrelationszeit $\tau_{\mathrm{c}}$ der Brownschen Bewegung und den Abstand $b$ zwischen Elektron und Proton in den Radikalen, sowie auf Naturkonstanten und die aus den benutzten Meßfrequenzen berechenbaren Kreisfrequenzen $\omega_{\mathrm{p}}$ und $\omega_{\mathrm{s}}$ zurückgeführt. Sie können berechnet werden, wenn $w_{\mathrm{s}}, \tau_{\mathrm{c}}$ und $b$ bekannt sind.

Im einzelnen sollte $b$ in dem hier interessierenden Phenylradikal, in dem das ungepaarte Elektron eine $\sigma$-Valenzeigenfunktion eines trigonalen Kohlenstoffs besetzt ${ }^{8}$, als Abstand von Elektron zu ortho-Proton etwa $2 \AA$ betragen, wie es die Geometrie des Radikals liefert. Für die Korrelationszeit $\tau_{\mathrm{c}}$ unserer Lösungen erscheinen nach der Literatur ${ }^{11}$ Werte von

10 F. J. Duncan u. A. F. Trotman-Dickenson, J. Chem. Soc. $1962,4672$.

11 E. R. Andrew, Nuclear Magnetic Resonance, Cambridge University Press, London 1956. 
$5 \cdot 10^{-12}$ sec bis $5 \cdot 10^{-11}$ sec vernünftig. Die Größe $w_{\mathrm{s}}$ läßt sich in unserem Fall nur schwer abschätzen. Sie beschreibt die Spin-Gitter-Relaxation der Elektronen bei Abwesenheit der dynamischen Kopplung mit den Radikalprotonen. Ein Hinweis auf den Wert von $w_{\mathrm{s}}$ läßt sich jedoch aus den bisher beobachteten niedrigsten Elektronenspinresonanzlinienbreiten kurzlebiger Radikale in Lösung von $\Delta H_{\mathrm{ms}} \approx 10^{+5}$ $\sec ^{-1}$ entnehmen ${ }^{12}$, die mit

$$
\Delta H_{\mathrm{ms}} \approx \frac{1}{T_{2 \mathrm{~s}}} \approx \frac{1}{T_{1 \mathrm{~s}}}>w_{\mathrm{s}}
$$

größer als $w_{\mathrm{s}}$ sein müssen.

Im besonderen ergeben sich nun mit dem aus a) bis d) gewählten Parametersatz:

$$
\begin{aligned}
B & =10^{-2} \mathrm{Mol} / l \cdot \mathrm{sec} ; \\
w_{\mathrm{s}} & =5 \cdot 10^{4} \mathrm{sec}^{-1} ; \\
n & =\int B \mathrm{~d} t=0,15 \mathrm{Mol} / l ; \\
\tau_{\mathrm{c}} & =2 \cdot 10^{-11} \mathrm{sec} ; \\
T_{1 \mathrm{M}} & =10 \mathrm{sec} \\
\tau & =5 \cdot 10^{-5} \mathrm{sec} ; \\
b & =2 \AA .
\end{aligned}
$$

aus den Gleichungen (6), (7), (10) und (15) für die verschiedenen Meßfrequenzen die in Tab. 1 angegebenen Verstärkungsfaktoren $V_{\text {theor }}$.

\begin{tabular}{llll}
\hline$v_{\mathrm{p}}[\mathrm{MHz}]$ & 40,0 & 56,4 & 100,0 \\
\hline$V_{\text {theor }}$ & $-9,5$ & $-6,6$ & $-1,5$ \\
$V_{\text {exp }}$ & -20 & $-8,5$ & $-2,9$ \\
\hline
\end{tabular}

Tab. 1. Berechnete und experimentelle Verstärkungsfaktoren.

Der Vergleich mit den ebenfalls angegebenen experimentellen Werten zeigt, da $\beta$ unsere Formeln den beobachteten Effekt in der Größenordnung richtig beschreiben. Da sie sicher nur näherungsweise gelten, scheint es nur wenig sinnvoll, durch Anpassung der bisher experimentell nicht exakt bestimmten sieben Parameter eine bessere als die erzielte Übereinstimmung zwischen Theorie und Experiment zu suchen.

\section{Folgerungen}

Die in dieser Arbeit angegebene Deutung der Protonenresonanzemissionslinien von Produkten rascher Radikalreaktionen des Typs

$$
\begin{aligned}
& \mathrm{R}-\mathrm{R} \rightarrow 2 \mathrm{R}^{\cdot}, \\
& \mathrm{R}^{\cdot}+\mathrm{L} \rightarrow \mathrm{M}+\mathrm{L}^{\circ}
\end{aligned}
$$

durch chemische induzierte dynamische Kernpolarisation behandelt nur die Molekeln M. Die Produkte weiterer Sekundärreaktionen, z. B. der Radikale L (vgl. ${ }^{1}$ ), wurden bisher nicht betrachtet. Sie könnten zu Emissionslinien Anlaß geben, wenn die Lebensdauern der Radikale $\mathrm{R}^{*}$ sehr kurz sind, und es ist leicht möglich, die oben angegebenen Überlegun gen auf diesen Fall, der auch bereits beobachtet wurde ${ }^{1}$ zu übertragen.

Weiterhin wird in dieser Arbeit eine mögliche Änderung der Kernresonanzspektren aller an den Reaktionen nicht unmittelbar beteiligten Molekeln der Lösungen, z. B. des Lösungsmittels, durch intermolekulare dynamische Kernpolarisation ${ }^{3,4}$ nicht berücksichtigt, da ein solcher Effekt bei unseren Experimenten nicht beobachtet wurde.

Der hier behandelte Befund sollte immer dann auftreten, wenn eine Reaktionsfolge vom Typ (1) und (2) in äußeren Magnetfeldern abläuft. Damit ist er nicht auf die bisher experimentell untersuchten thermischen Spaltungen von Peroxiden oder Azoverbindungen beschränkt, sondern sollte auch bei photolytischer oder radiolytischer Radikalerzeugung beobachtet werden. Sie sollten insbesondere die Möglichkeit bieten, die Emissionssignale in einem stationären Zustand der Reaktionen- und Relaxationsfolge zu beobachten und damit die Gültigkeit der hier entwickelten Gleichungen genauer zu prüfen, als es bisher möglich war.

Schließlich lassen sich die hier durchgeführten Überlegungen auch auf den Fall übertragen, daß nicht nur die Dipol-Dipol-Kopplung, sondern auch die skalare Kopplung zwischen Elektronen- und Kernspinmomenten zeitlich moduliert ist. In diesem Fall sollten anstatt intermediärer Emissionslinien überhöhte Absorptionslinien beobachtet werden, da $\sigma$ hier negativ werden kann $^{3}$ [vgl. Gl. (15)]. Tatsächlich wurden solche Absorptionslinien während einiger chemischer Reaktionen bereits von uns beobachtet. Sie werden in späteren Arbeiten publiziert.

Wir danken Herrn Professor Dr. K.-H. Hellwege und Herrn Priv.-Doz. Dr. U. Johssen für Diskussionen und die Unterstützung beim Fortgang dieser Arbeit. Besonderen Dank schulden wir Herrn Professor Dr. K. H. Hausser, Heidelberg, für eingehende Diskussionen und seine Hilfe bei der Deutung des Effekts.

12 R. W. Fessenden u. R. H. Schuler, J. Chem. Phys. 39, 2147 [1963]. 